\title{
SUL MEDESIMO E L'ALTRO. TRA FOLLIA E LETTERATURA NELLA PROSPETTIVA SEMIOTICA DI STUDI DI MICHEL FOUCAULT ${ }^{1}$
}

\author{
ON THE SAME AND THE OTHER. BETWEEN MADNESS \\ AND LITERATURE \\ FROM THE SEMIOTIC PERSPECTIVE OF STUDIES \\ BY MICHEL FOUCAULT
}

\begin{abstract}
Filippo SILVESTRI
Università degli Studi di Bari “Aldo Moro"

filippo.silvestri@uniba.it
\end{abstract}

Riassunto: Il rapporto tra il Medesimo e l'Altro rappresenta un leit motiv lungo l'intero arco della ricerca di Foucault. Negli anni Sessanta egli ha certamente tematizzato il problema, affrontandolo da due prospettive. L'altro è tutta la storia della follia dalla fine del Medioevo fino al limite dei primi studi di Freud. L'altro è la scrittura letteraria di Sade, Hölderlin, Nietzsche, Artaud, Bataille, Roussell, Blanchot, Klossowski, tutti insieme convocati su uno scenario filosofico, che si muove ad elastico tra teorie surrealiste della scrittura e dell'opera d'arte e prime forme di uno strutturalismo in pieno svolgimento.

Parole chiave: Medesimo. Altro. Letteratura. Follia. Foucault.

Abstract: The relationship between the Self and the Other represents a leitmotiv throughout Foucault's research. In the sixties, he certainly addressed the issue from two perspectives. The Other is the whole history of madness from the end of the Middle Ages to the limits of Freud's early studies. The Other is in the literary writings by Sade, Hölderlin, Nietzsche, Artaud, Bataille, Roussell, Blanchot, Klossowski, who are all summoned together on a philosophical scenario which ranges from surrealist theories of writing, and the work of art and first forms of structuralism in full swing. 
Key Words: Same. Others. Literature. Madness. Foucault.

\section{BREVE INTRODUZIONE AI PROBLEMI}

Certamente Michel Foucault non è stato un semiotico o se si vuole, come è noto, nemmeno un filosofo del linguaggio: affermazioni queste che sanno di paradossale, se solo si considerano due opere su tutte, Le parole e le cose (Foucault, 1998) e Archeologia del sapere (Foucault, 1999), che sono piene di riferimenti al problema del linguaggio, nelle sue diverse varianti fondative, in senso archeologico così come intende il problema "archeologico" sempre Michel Foucault. Ma tant'è: mai nelle pagine del filosofo francese, fin dall'inizio con Storia della follia nell'età classica (Foucault, 2004) non c'è mai (dicevamo) una dichiarazione aperta di natura genetico/costitutiva, che dica in modo netto di un carattere fondativo della faglia semiotica delle cose, sempre invece a vario titolo intrecciata ad altri fenomeni genericamente di natura storica (quindi politici, sociologici, antropologici, in senso lato culturali) altrettanto decisivi ed importanti nella definizione di un inizio e di una fine di un'epoca, secondo una disposizione delle cose nel corso del tempo, per cui per Foucault ogni epoca dell'immagine del mondo - il riferimento è ovviamente qui ad Heidegger (1984) - ha il suo apriori storico, dove questa storicità non è solo e principalmente ed in primo luogo semiotica.

Questioni di impostazioni della ricerca. Colpisce, almeno noi che scriviamo, che proprio in Italia negli anni Settanta Carlo Sini (1978: 543-558) abbia rivolto un atto di accusa simile ad un altro filosofo/non filosofo del linguaggio, di un'altra generazione rispetto a Michel Foucault, Edmund Husserl, a cui rimproverava quello che in certo modo stiamo rimproverando noi a Foucault, di non essere stato abbastanza semiotico nei suoi presupposti filosofici, non almeno tanto quanto lo era stato a suo tempo Charles Sanders Peirce. Colpisce sempre e allo stesso modo come Enrico Redaelli (2011) sempre in Italia abbia rivolto allo stesso Foucault la stessa obbiezione ovvero di non essere stato abbastanza semiotico, se non altro almeno come sempre lo stesso Peirce era stato: accuse tutte oggi legittime, in quanto messe in luce di limiti essenziali dell'impostazione della ricerca da parte di due giganti come Husserl e Foucault, non abbastanza peirciani, entrambi e per diverse ragioni, lungo vie sostanzialmente differenti. 


\section{LA SEMIOTICA DI MICHEL FOUCAULT E I SUOI DI PIÙ}

Ma limitiamoci qui a Foucault. Detto che effettivamente questa mancanza o per meglio dire questo sbilanciamento semiotico non c'è stato, ripetiamo quanto detto all'inizio ovvero che non si può altrettanto clamorosamente negare una sua sensibilità (di Michel Foucault) dall'inizio alla fine delle sue ricerche al problema (fondativo) del linguaggio, sia in senso archeologico che genealogico, cosa per altro riconosciuta ultimamente da almeno un paio di studi monografici, dedicati ad un confronto niente affatto fuori luogo proprio tra Foucault e Wittgenstein (Gros, Davidson, 2011; Gillott, Lorenzini, 2016). Detto molto in breve e per ragioni di spazio e di equilibrio in questo nostro discorso, Foucault sta insieme con Wittgenstein su un comune terreno pragmatico, in cui le pratiche discorsive (così centrali in Archeologia del sapere) hanno un ruolo fondativo rispetto alle società, le comunità che si raccolgono insieme intorno a quei linguaggi e che si distinguono nel corso del tempo nelle diverse epoche della storia proprio a partire dai modi con cui giocano con il linguaggio, per dire le cose che si possono dire e quelle che invece vanno assolutamente taciute. Ovviamente per Foucault la cosa, come è noto, non si riduce ad una sola questione linguistica o più in generale semiotica, se proprio in Archeologia del sapere (1999) si ritrova ad un certo punto a scrivere, puntualizzando:

[...] vorrei mostrare, con esempi precisi, che, analizzando i discorsi stessi, si vede allentarsi la stretta apparentemente tanto forte delle parole e delle cose, e farsi luce un insieme di regole proprie della pratica discorsiva. [...] "Le parole e le cose" è il titolo [di...] un compito che consiste nel non trattare —nel non trattare più- $i$ discorsi come degli insiemi di segni (di elementi significanti che rimandino a contenuti e a rappresentazioni), ma come delle pratiche che formano sistematicamente gli oggetti di cui parlano. Indubbiamente $i$ discorsi sono fatti di segni; ma fanno molto di più che utilizzare questi segni per designare le cose. È questo di più che li rende irriducibili alla langue $e$ alla parole. È questo di più che bisogna mettere in risalto e bisogna discutere (Foucault, 1999: 66-67).

Questo di più è ancora una volta il Foucault semiotico che non è 
semiotico, che non è solo un semiotico, ma molto altro ancora ed insieme, in un continuo tira e molla con 'la cosa in sé semiotica', che lui avvicina e che allontana. In ogni caso, che le cose stiano così ovvero che ci sia un Foucault molto pragmatico nel riconoscere un ruolo fondativo al linguaggio, sembra cosa per noi confermata da due diverse circostanze in due diversi momenti della storia delle sue ricerche. Le direzioni verso le quali stiamo guardando sono esplicitamente segnate da due momenti filosofici foucaultiani ovvero da Sorvegliare e punire (Foucault, 1993) e da Le confessioni della carne (Foucault, 2019), quest'ultimo uscito recentemente nell'edizione francese (Foucault, 2018), subito seguita a stretto giro di un anno dalla traduzione italiana (Foucault, 2019). Insomma e per andare dentro il problema e per fare solo due esempi tra i molti altri possibili², è noto come in Sorvegliare e punire la disposizione panottica del carcere di Bentham sia decisiva e determinante da un punto di vista architettonico e dunque semiotico per Foucault, per capire come si sorveglia, come si punisce, come si controlla, come si è controllati all'interno di un sistema punitivo. Molto diversamente, ma sempre in senso semiotico, è una precisa pratica discorsiva, la "confessione", a determinare i modi delle relazioni possibili in una comunità intorno a ciò che è buono e a ciò che non è buono, se si vuole fondare un giusto rapporto nel vivere civile comune.

\section{FOUCAULT E L'ALTRO COME TESTO LETTERARIO}

Ma fatto quest'ordine di considerazioni che vale alla stregua di una sorta di giustificazione semiotica per la nostra discussione, c'è stato un tempo, gli anni Sessanta, in cui Michel Foucault è stato più decisamente 'semiotico' rispetto ad altri momenti della sua ricerca e con questo stiamo facendo riferimento a tutta una serie di cosiddetti "scritti letterari" (Foucault, 2004), in cui per davvero e stando nei temi di questo convegno, Michel Foucault ha discusso il problema del Medesimo e dell'Altro, dell'alterità per usare un'espressione levinassiana, proprio a partire da un orizzonte di studi semiotico perché appunto letterario. Detto

\footnotetext{
${ }^{2} \mathrm{Si}$ pensi, solo per fare un esempio che diverge da quelli letterari che discuteremo, a tutta l'attenzione che Michel Foucault ha dedicato alla pittura, ai grandi pittori, in tanti momenti diversi della sua ricerca filosofica da Goya (Foucault, 2004a: 448-449) a Manet (Foucault, 2004c), a Magritte (Foucault, 1973).
} 
in estrema sintesi negli anni Sessanta per Michel Foucault la letteratura ha rappresentato la vera (non certo l'unica) alternativa al discorso scientifico delle identità affermate, per cui si può e si deve arrivare a dire/scrivere $S$ è $p$ : le scritture letterarie ${ }^{3}$, in modo particolare quelle di Maurice Blanchot e Raymond Roussell, non molto diversamente ma su prospettive e piani differenti rispetto ai due appena citati invece nell'Ottocento Sade ed Holderlin, ma ancora insieme di nuovo nel Novecento Bataille, Klossowski, rappresentano tutti in senso semiotico una possibilità di dire diversamente il mondo della vita che sottendono (Husserl) e che sta alla base degli stessi discorsi scientifici, altrimenti possibili appunto su piani semiotici differenti. Valga su tutto proprio l'apertura della prefazione a Le parole e le cose, dove appoggiandosi a Luis Borges, al Borges di L'idioma analitico di John Wilkins (Borges, 1963), Michel Foucault scrive quanto segue a proposito di come e quanto la "letteratura" possa essere ascrivibile ad un ordine del discorso che non è quello del Medesimo ma dell'Altro e proprio quando si tratta di categorizzare le cose:

Questo libro [Le parole e le cose] nasce da un testo di Borges: dal riso che la sua lettura provoca, scombussolando tutte le familiarità del pensiero - del nostro, cioè: di quello che ha la nostra età e la nostra geografiasconvolgendo tutte le superfici ordinate e tutti $i$ piani che placano ai nostri occhi il rigoglio degli esseri, facendo vacillare e rendendo a lungo inquieta la nostra pratica millenaria del Medesimo e dell'Altro. Questo testo menziona "una certa enciclopedia cinese" in cui sta scritto che "gli animali si dividono in: a) appartenenti all'Imperatore, b) imbalsamati, c) addomesticati, d) maialini da latte, e) sirene, f) favolosi, g) cani in libertà, h) inclusi nella presente classificazione, i) che si agitano follemente, $j$ ) innumerevoli, $k$ ) disegnati con un pennello finissimo di peli di cammello, l) et caetera, $m$ ) che fanno l'amore, $n$ ) che da lontano sembrano mosche". Nello stupore di questa tassonomia, ciò che balza subito alla mente, ciò che, col favore dell'apologo, ci viene indicato come il fascino esotico d'un altro pensiero, è il limite del nostro, l'impossibilità pura e semplice di pensare tutto questo (Foucault, 1998: 5).

\footnotetext{
${ }^{3}$ Particolarmente interessante a nostro avviso la circostanza per cui lo stesso Algirdas Julien Greimas nel suo Dell'imperfezione (Greimas, 2004) concluda il suo percorso accademico proprio con un'apertura nei confronti della soluzione letteraria (Calvino, Tournier, Rilke, Tanizaki, Cortázar) come alternativa ad una semiotica altrimenti costituita e luogo possibile per una rappresentazione dell'ineffabile in senso estetico.
} 
Solo per restare in un ambito semiotico di considerazioni, proprio a proposito di cosa significa categorizzare le cose, come si possono categorizzare le cose, è certamente noto come John R. Taylor (1995) con il suo Linguistic categorization: prototypes in linguistic theory, Umberto Eco (1997) e Patrizia Violi (1997) due anni dopo rispettivamente con Kant e l'ornitorinco e con Significato ed esperienza, abbiano posto in tempi ancora per noi assolutamente attuali, almeno per noi in Italia, il problema della fondazione semiotica del rapporto con le esperienze delle cose in una stretta relazione con la questione concorrenziale delle categorizzazioni del pensiero. Qui, in questo incipit di Le parole e le cose, con consapevolezza e propositi autoironici Foucault si dice e dice al suo lettore come sia difficile, ai limiti dell'impossibile il progetto che lui stesso si è proposto ovvero quello di stabilire un rapporto tra le parole e le cose ovvero ed ancora il rapporto tra come si dicono le cose e come uno/a ne fa un'esperienza in primo luogo percettiva, soprattutto guardandole, ferma restando la grande lezione di Maurice Merleau-Ponty (1964), quella di Le visible et l'invisible che per Foucault sarà allo stesso modo e diversamente 'il dicibile, l'indicibile', ferma restando un'altra lezione questa volta di Wittgenstein a proposito delle cose di cui si deve tacere e quelle invece di cui si può parlare. Insomma e per tornare a Le parole e le cose il progetto di Foucault naufraga, ironia delle sorte, proprio sul limite del suo incipit, naufraga di fronte all'enciclopedia impossibile e cinese raccontata da Luis Borges, naufraga di fronte al gesto della parola e della scrittura letteraria che si spostano di lato e lasciano cadere fuori dalle loro righe un programma di netta definizione di cosa sia il "medesimo" e "l'altro". Una pretesa categoriale del genere collassa di fronte al gesto letterario, sempre possibile, che enumera all'infinito le infinite possibilità per una definizione categoriale. Tutto questo per Foucault è possibile, perché viviamo la nostra vita proprio ed ancora a partire da un linguaggio che quella vita può rappresentare 'sempre di nuovo' (immer wieder, di nuovo Husserl ed insieme a lui Merleau-Ponty). Scrive Foucault:

Ciò che è impossibile, non è la vicinanza delle cose, ma il sito medesimo in cui potrebbero convivere. Gli animali "i) che si agitano follemente, $j$ ) innumerevoli, k) disegnati con un pennello finissimo di peli di cammello" dove potrebbero incontrarsi, se non nelle voce immateriale che ne pronuncia l'enumerazione, se non sulla pagina che la trascrive? Dove possono giustapporsi se non nel nonluogo del linguaggio? (Foucault, 1998: 6). 


\section{FOUCAULT E L'ALTRO TRA LETTERATURA E FOLLIA}

Il problema dell'altro, dell'altra negli anni Sessanta della ricerca di Michel Foucault, è discusso ed ipotizzato a partire da due diverse istanze costitutive del senso delle parole e delle cose ovvero a partire da due istanze che non sono necessariamente fondative della costituzione di un senso, se questo viene inteso in modo banalmente scientifico, ammesso e non concesso che ci possa mai essere una scienza che come tale sia banale: si tratta dell'esperienza della follia così come è stata pensata, vissuta ed organizzata dal Cinquecento in poi (Storia della follia nell'età classica [Foucault, 2004a]), si tratta diversamente dell'esperienza letteraria così come è stata vissuta/interpretata da Sade ed Hölderlin, da Antonin Artaud, da Bataille e Klossowski, da Raymond Roussel, da Maurice Blanchot. Questi i due diversi piani lungo i quali Michel Foucault negli anni Sessanta conduce la sua indagine a cavallo tra le due dimensioni (non dimensioni) del Medesimo e dell'Altro, secondo due diverse possibili divergenze rispetto agli ordini del discorso costituiti e vigenti ovvero e rispettivamente l'esperienza della follia da una parte e della letteratura dall'altra, all'atto pratico e storico, almeno da Sade ed Holderlin, Nietzsche in poi, fino al limite della loro convergenza, della convergenza dell'esperienza della follia con quella della letteratura. Scrivere fino ad impazzire, impazzire e dunque scrivere, essere considerati folli e dunque liberi di dire/scrivere quello che uno/a vuole dire, scrivendo poesie, romanzi, aforismi, genealogie della morale, con il martello in mano, il martello che adoperano prima Sade, poi Nietzsche, Sade su quel confine, di fronte a quel limite in cui la letteratura intercetta e diventa un'esperienza erotica non riconosciuta, inaccettabile, non ordinabile, inconfessabile, oscena e degna di una prigione ovvero ancora un'esperienza erotica che deve essere confessata, che deve essere detta, che deve essere tradotta in un discorso, in una psicoanalisi che la comprenda, che la curi, che la renda accettabile agli occhi, alle orecchie dei più. Scrivere fino ad impazzire, scrivere perché si è impazzati: d'amore e non solo. Foucault in una famosissima intervista intitolata poi almeno nella raccolta italiana Follia, letteratura, società (siamo nel 1970) ad un certo punto almeno a partire dalla trascrizione dirà: 
Oggi non si può intraprendere la strana esperienza della scrittura senza affrontare il rischio della follia. È ciò che Hölderlin e, in una certa misura, Sade ci hanno insegnato. Secondo me si può dire la stessa cosa della filosofia. [...] Ora, con Nietzsche giunge finalmente questo momento in cui la filosofia dirà: "Alla fine, può darsi che io sia folle". [...] Su questo punto la scrittura successiva al XIX secolo esiste manifestamente per se stessa e, se necessario, esisterebbe indipendentemente da ogni consumo, da ogni lettore, da ogni piacere e da ogni utilità. Ora, questa attività verticale e quasi intrasmissibile della scrittura somiglia in parte alla follia. In un certo senso, la follia è un linguaggio che si mantiene verticale, e che non è più la parola trasmissibile, avendo perduto ogni valore di scambio. Sia perché la parola ha perduto ogni valore e non è desiderata da nessuno, sia perché si esita a servirsene come di una moneta, come se le fosse attribuito un valore eccessivo. Ma, in fin dei conti, i due estremi si toccano. Questa scrittura che non circola, questa scrittura che sta in piedi è proprio un equivalente della follia. È normale che gli scrittori trovino il loro doppio nel folle o in un fantasma (Foucault, 2014: 270-271).

Non è solo questione di questo strano confine tra follia e letteratura, come altrettanto bene se si legge Storia della follia nell'età classica tra follia e pittura (si pensi al Narrenschift di Bosch, ancora ai Goya che chiudono Storia della follia nell'età classica [Foucault 2004a: 448449]), ad interessare ed impegnare nei suoi studi Michel Foucault negli anni Sessanta, ripetiamo a stretto giro di un confronto sempre latente in senso epistemologico, ma già in sé secondo modi che sono della politica, discutendo sempre il rapporto che passa tra il Medesimo e l'Altro. È la follia che lo coinvolge nei primi passi della sua ricerca, la follia nelle sue diverse manifestazioni nel corso della storia recente, che dal Medioevo va fino dentro i primi studi di Sigmund Freud. È la letteratura in sé e per sé ad interessarlo, come testimoniato dalla raccolta Scritti letterari cui abbiamo già fatto riferimento e sulla quale torneremo a breve, come testimoniano d'altra parte un'altra serie di interventi di Michel Foucault di quegli stessi anni, per la precisione due conferenze alla radio tenute a Parigi nel gennaio del 1963 e due conferenze tenute a Bruxelles nel dicembre del 1964 ed una conferenza tenuta all'Università di Buffalo nel 1970, oggi tutte raccolte in un volume intitolato significativamente La grande étrangère. À propos de littérature (Foucault, 2013). I titoli forse meglio di qualsiasi altra cosa riassumono i temi ricorrenti nel pensiero di Foucault di quegli anni: Le langage de la folie, Littérature et langage, Conférences su Sade. Noi 
resteremo per questioni di spazio agli Scritti letterari ed in particolare al famoso Il pensiero del fuori dedicato alla scrittura di Maurice Blanchot, lavoro del giugno del 1966, sempre dunque in quegli stessi anni.

\section{IL FUORI DI MAURICE BLANCHOT}

Che cos'è questo "fuori" di Blanchot, di Foucault? Fuori da che cosa, rispetto a chi? I passaggi per arrivare ad una spiegazione sono vari ed impervi e dobbiamo procedere con attenzione (Artieres, Gros, Revel e Gaillard, 2004; Iacomini, 2008: 246-253; Favreau, 2012: 201-256). Tutte le complicazioni che dovremo provare a sciogliere si raccolgono nel nodo di diversi fili, il filo della sovranità del linguaggio, quello della nozione di "assenza di opera", il filo della "cancellazione della soggettività" (del depotenziamento del soggetto), in nome tutto di un superamento del limite (quale limite, forse quello del medesimo, del soggetto, dell'interiorità, del dentro del soggetto?), in nome di una contestazione che facciamo sempre una certa fatica con Foucault a non considerare anche politica e non solo dunque letteraria. A confronto nelle pagine di Maurice Blanchot (2018: 179-184) rilette da Foucault, due diversi eroi della classicità, Orfeo ed Ulisse, tutti e due alla prova della tentazione dell'immagine, della voce, del desiderio, dell'erotico, di Euridice e delle Sirene: due diversi esiti di questi incontri, di questi momenti che sono della tentazione, della trasgressione (Bataille). Orfeo non resisterà, si girerà, perdendo Euridice, uscendo di sé, assecondando il suo desiderio, dissipando soprattutto un lavoro faticoso, l'opera di recupero di Euridice, sostenuta dagli dei, sebbene a certe condizioni. Orfeo è il dissipatore della sua opera perché cede al desiderio. Ulisse no, non cede al suo desiderio: legato all'albero della nave sente il canto delle sirene ma non si lascia andare, non dissipa la sua opera, quella del ritorno a sé, alla sua patria, ad Itaca. Resta in sé, non dà di fuori, non dà di matto, non esce fuori dalle righe del suo ego cogito. E tuttavia la sua astuzia, l'astuzia di Ulisse per Maurice Blanchot "si addice ad un Greco della decadenza che non meritò di essere l'eroe dell'Iliade" (Blanchot, 2019: 12).

Che la questione sia complessa è un dato di fatto, perché a confronto sono due modelli. Concentriamoci su uno di loro, su Orfeo. Quello che Orfeo fa non è utile, non è produttivo di nulla (non produce un'opera), non consente ad Orfeo di riprendere se stesso: tantomeno Euridice. La sua 
scelta non scelta non lo porta a nulla: viene dal nulla del mondo dei morti, finisce nel nulla della sua opera (non opera) incompiuta. Dal nulla al nulla. Orfeo non ha un progetto: la sua identità di soggetto non esce fortificata dall'esperienza che fa. Il suo voltarsi indietro è dimenticanza, è "oblio": dimentica quello che avrebbe dovuto fare per portare a compimento l'opera. Cede al "fuori", cede all'esterno, cede al desiderio e non gli torna indietro nulla. Il gesto di Orfeo, quello di voltarsi indietro, ha il tipico carattere improduttivo del gesto letterario, che non punta alla produzione di un'opera, perché è pura dépense ovvero con le parole di Wanda Tommasi è leggerezza, indifferenza, innocenza, futilità, pura perdita, circostanze tutte queste che ci mettono "in prossimità del sacro" (Tommasi, 1984: 61-65)4. In modo per noi complesso, complicato, ma con accenni che possiamo comprendere, Foucault scrive:

Al momento in cui l'interiorità è attirata fuori di sé, un di fuori scava il luogo stesso dove l'interiorità ha l'abitudine di trovare il suo ritiro e la possibilità del suo ripiegamento: sorge una forza -meno di una forma, una sorta di anonimato informe e testardo - che spodesta il soggetto della sua semplice identità [...] lo spodesta del suo diritto immediato a dire Io ed eleva contro il suo discorso una parola che è indissolubilmente eco e diniego (Foucault, 2004b: 128).

Come non rilevare in queste parole di Foucault tutta la complessità del suo rapporto con Maurice Blanchot (ed ancora con Bataille), tutta la complicazione di un pensiero che cerca di mostrare, nominare, additare cose che non sono cose, dimensioni che non sono dimensioni, un altro rispetto al medesimo che sono io. Ma forse nemmeno: con Foucault insieme a Maurice Blanchot bisogna come provare a collocarsi oltre la differenza tra il Medesimo e l'Altro, in un territorio desolato, disumano, desertico. Di qui la necessità di cedere di nuovo la parola a Foucault, per fargli dire quello che vuole dire, leggendo Blanchot, leggendo Roussell, Bataille, leggendo, scrivendo di letteratura:

\footnotetext{
${ }^{4} \mathrm{Si}$ veda inoltre come Miriam Iacomini (Iacomini, 2008: 247) metta in evidenza una certa differenza intuita dalla stessa Wanda Tommasi tra le posizioni di Bataille e quelle di Blanchot, entrambi punti di riferimento di Foucault negli anni Sessanta, perché mentre in Bataille la dépense è a trecentosessanta gradi, in Blanchot sussiste ancora una tensione tra padronanza ed eccesso, tra lavoro e dispendio.
} 
Tendere l'orecchio verso la voce argentata delle Sirene, rivoltarsi verso il volto proibito che già si è sottratto alla vista, non è soltanto infrangere la legge per affrontare la morte, non è soltanto abbandonare il mondo e la distrazione dell'apparenza, è sentire improvvisamente crescere dentro di sé il deserto nel quale, all'altra estremità (ma questa distanza senza misura è anche sottile quanto una linea), balena un linguaggio senza l'assegnazione di un soggetto, una legge senza Dio, un pronome personale senza personaggio, un volto senza espressione e senza occhi, un altro che è il medesimo (Foucault, 2004b: 128).

Un altro che è il medesimo, un deserto che cresce all'improvviso dentro di me: soprattutto, per noi che stiamo rincorrendo Foucault lungo le vie impervie della sua semiotica mancata, un linguaggio senza che gli sia assegnato un soggetto. Difficile dire con certezza che cosa Foucault intenda con quest'ultima espressione: un linguaggio senza l'assegnazione di un soggetto non è forse quel linguaggio, struttura disumana, morte dell'uomo che chiude Le parole e le cose? E che cosa ha a che fare questo linguaggio con lo strutturalismo di cui Foucault è contemporaneo ed interprete nella sua Parigi degli anni Sessanta? I passaggi sono complicati, perché è come se si tendesse un arco ampio che va dalla letteratura (almeno da Blanchot, Roussell, Bataille, Klossowski, Sade, Hölderlin) fino allo strutturalismo, fino a La filosofia strutturalista permette di diagnosticare che cos'è "oggi" (Foucault, 2014: 147-152), attraverso percorsi diversi che conducono ad uno stesso esito, in quegli anni: ad un linguaggio in cui l'uomo non si trova ma si perde, ad un linguaggio che è in larga parte alla stregua di una possibilità semiotica alternativa e dunque dalla parte della letteratura, ad un linguaggio struttura in cui l'uomo non è più il soggetto cartesiano dominante, non è più una parole decisiva per la vita della langue. Due passaggi diversi, entrambi semiotici, verso un esito comune: la scomparsa dell'uomo o ancora, non molto diversamente, la fine copernicana della sua presunta centralità, materia tra la materia o ancora per essere nicciani, heideggeriani, forza tra le forze, un esserci nell'apertura dell'Essere. Foucault (2004b: 119): “L'attrazione è per Blanchot quello che probabilmente per Sade è il desiderio, per Nietzsche la forza, per Artaud la materialità del pensiero, per Bataille la trasgressione: vale a dire l'esperienza più pura e più scarna del di fuori'. Di certo non più l'ego cogito della fenomenologia husserliana al centro del 
pensiero filosofico francese di quegli anni, ma nemmeno l'esistenza così come la intendeva allora già da qualche anno Sartre, oltre le dinamiche del Medesimo, dell'Altro, perché ricorda Miriam Iacomini: "Teorizzare, infatti, la sovranità del linguaggio implica, come ben dimostrano i romanzi dell'avanguardia post-surrealista, un depotenziamento del soggetto che diventa semplicemente elemento in balia di una esteriorità che lo estirpa dalla propria interiorità" (Iacomini, 2008: 253).

Di sicuro con Blanchot (non diversamente con Roussell ed un po' con tutti gli altri sempre citati) siamo dentro le maglie ben strutturate di un linguaggio letterario che non ha più una funzione referenziale: non è strumentale, non è uno strumento nelle mani dell'uomo. Siamo insieme al Foucault degli anni Sessanta nel guado di una scrittura che è dell'attimo, che si consuma nel momento del gesto di chi scrive o prende la parola, una scrittura poetica in cui non ci deve essere per forza di cose un rapporto tra significante e contenuto (Iacomini, 2008: 251): un piacere del testo (Barthes, 1999) ovvero un testo che cede al piacere, al desiderio (come fa Orfeo), all'attrazione per il fuori, all'attrazione per il vuoto, un testo che viene dal vuoto e va verso il vuoto, un vuoto che non è umano in nessuno dei due casi. Oltre un problema sempre politico e per tanti versi inevitabilmente semiotico, oltre il problema del rapporto dialettico e non dialettico tra il Medesimo e l'Altro vale all'inizio della nostra epoca moderna l'annuncio nicciano, foucaultiano della morte dell'uomo:

Più che la morte di Dio - o meglio nella scia di tale morte e in una correlazione profonda con essa il pensiero di Nietzsche annuncia la fine del suo uccisore: ossia l'esplosione del volto dell'uomo nel riso, e il ritorno delle maschere; la dispersione della profonda colata del tempo da cui l'uomo si sentiva parlato e di cui sospettava la pressione nell'essere stesso delle cose; l'identità tra il Ritorno del Medesimo e l'assoluta dispersione dell'uomo. Durante l'intero XIX secolo, la fine della filosofia e la promessa d'una cultura prossima coincidevano probabilmente con il pensiero della finitudine e l'apparizione dell'uomo nel sapere; oggi, il fatto che la filosofia sia sempre e ancora sul punto di scomparire, e il fatto che forse in essa, ma più ancora fuori di essa e contro di essa, nella letteratura come nella riflessione formale, si pone il problema del linguaggio, dimostrano probabilmente che l'uomo sta sparendo (Foucault, 1998: 412). 


\section{PER CONCLUDERE. LE PAROLE DEL FOLLE}

Ma forse per provare a chiudere questo nostro lavoro è ancora necessario un passo indietro che vada insieme a quello letterario che abbiamo cercato di fare, un passo di lato, indietro e di lato, in direzione di nuovo della follia, del linguaggio della follia, ammesso e non ancora concesso che alla follia, ai folli sia concessa la parola. Chi è questo folle a cui è concesso di parlare ed in quale contesto? Foucault è esplicito: c'è stato un tempo in cui la follia poteva parlare, aveva un ruolo, addirittura quello di dire la verità, sulle tavole di un palcoscenico, di fronte alla platea di un teatro, in un tempo, un momento della giornata che era del gioco e della festa. La parola del folle era allora una parola di verità, detta e pronunciata nel tempo della sospensione giocosa delle cose umane (Foucault, 2014: 268-270). Nel XIX secolo con Sade e Hölderlin la parola del folle sarà re-interpretata dalla letteratura e nella dimensione letteraria troverà la sua legittimazione, nel senso dei luoghi e dei tempi legittimi per una sua possibile esposizione pubblica, sebbene la stessa fosse ancora allora una parola scandalosa perché erotica e dunque degna di una prigione (Sade), sebbene sempre allora fosse una parola che era del poeta in un momento del suo impazzimento (Hölderlin, mai dimenticando l'esito della vita di Nietzsche). In tempi non sospetti, perché precedenti lo spartiacque delle Meditazioni cartesiane ${ }^{5}$, la parola è stata del folle sulle scene del teatro, quando al folle era concesso parlare, quando al folle in particolare si riconosceva addirittura la prerogativa di dire la verità fuori dalle righe, fuori dell'ordine del discorso socialmente riconosciuto. Poi nel corso del tempo il gioco delle sovrapposizioni ha complicato il rapporto tra il Medesimo e l'Altro, fino a far coincidere anche se solo in controluce ciò che è stato considerato folle con la verità profonda del nostro pensiero contemporaneo. Foucault in La Follia, l'assenza d'opera, scriverà:

Forse, un giorno, non sapremo più esattamente che cosa ha potuto essere la follia. La sua figura si sarà racchiusa su se stessa non permettendo più di decifrare le tracce che avrà lasciato. Queste stesse tracce non appariranno,

\footnotetext{
${ }^{5}$ Secondo il Foucault di Il mio corpo, questo foglio, questo fuoco Cartesio escluderà con decisione la follia dal suo ordine del discorso perché troppo destabilizzante rispetto a tutto l'impianto filosofico complessivamente considerato: se ammetto la possibilità di essere folle, a collassare è l'intero castello del cogito ergo sum (Foucault, 2004a: 485-509).
} 
a uno sguardo ignorante, se non come semplici macchie nere? Tutt'al più faranno parte di configurazioni che a noi sarebbe impossibile disegnare, ma che saranno nel futuro le indispensabili griglie attraverso le quali render leggibili noi, e la nostra cultura, a noi stessi. Artaud apparterrà alla base del nostro linguaggio, e non alla sua rottura; le nevrosi, alle forme costitutive (e non alle deviazioni) della nostra società. Tutto quel che noi oggi proviamo relativamente alla modalità del limite, o della estraneità, o del non sopportabile, avrà raggiunto la serenità del positivo. E quel che per noi designa attualmente questo Esterno rischia veramente un giorno di designarci, noi proprio noi (Foucault, 2004a: 475).

Così e davvero per concludere nello stesso luogo semiotico in cui si discrimina tra ciò è vero e ciò che è falso, tra ciò che è mio e ciò che è tuo, stabilendo dove sta il Medesimo (io) e l'altro (il tu), nella stessa piega semiotica delle cose convergono due diversi momenti della semiotica foucaultiana, quello della parola del folle sulle tavole di un palcoscenico da dove dice la verità, quello del parressiasta (Foucault, 2016) che il Foucault delle ultime lezione al College de France convoca sulla scena politica della Grecia e della Roma classiche, per dire cinicamente la verità al sovrano, al re che potrà punirlo, un cinico allora non ancora certamente 'folle' ma altro perché 'filosofo' rispetto al medesimo del potere, che è un potere di vita e di morte.

\section{RIFERIMENTI BIBLIOGRAFICI}

ARTIERES, P.; GROS, F.; REVEL, J. e GAILLARD, F. (eds.) (2004). Michel Foucault, la littérature et les arts: Actes du colloque de Cerisy - juin 2001. Paris: Kimé.

BARTHES, R. (1999). Variazioni sulla scrittura, seguite da Il piacere del testo. Torino: Einaudi.

BLANCHOT, M. (2018). Lo spazio letterario. Milano: Il Saggiatore. (2019). Il libro a venire. Milano: Il Saggiatore.

BORGES, J. L. (1963). Altre inquisizioni. Milano: Feltrinelli.

ECO, U. (1997). Kant e l'ornitorinco. Milano: Bompiani.

FAVREAU, J. F. (2012). Vertige de l'écriture. Michel Foucault et la littérature (1954-1970). Lyon: Ens Éditions.

FOUCAULT, M. (1973). Ceci n'est pas une pipe. Montpellier: Éditions 
Fata Morgana.

(1993). Sorvegliare e punire. Torino: Einaudi.

(1998). Le parole e le cose. Un'archeologia delle scienze umane.

Milano: BUR.

(1999). L'archeologia del sapere. Una metodologia per la storia della cultura. Milano: BUR:

(2004a). Storia della follia nell'età classica. Milano: BUR.

(2004b). Scritti letterari. Milano: Feltrinelli.

(2004c). La peinture de Manet. Paris: Éditions du Seuil.

(2013). La grande étrangère. À propos de littérature. Paris: EHESS. (2014). Follia e discorso. Archivio Foucault 1. Interventi, colloqui, interviste. 1961-1970. Milano: Feltrinelli.

(2016). Il coraggio della verità. Il governo di sé e degli altri II. Corso al Collège de France. Milano: Feltrinelli.

(2018). Les aveux de la chair, Histoire de la sexualité 4. Paris: Éditions Gallimard.

(2019). Le confessioni della carne. Milano: Feltrinelli.

GILLOT, P. et LORENZINI, D. (2016). Foucault Wittgenstein. Subjectivité, politique, étique. Paris: CNRS Éditions.

GREIMAS, A. J. (2004). Dell'imperfezione. Palermo: Sellerio Editore Palermo.

GROS, F. et DAVIDSON A. (2011). Foucault, Wittgenstein: de possible rencontres. Paris: Éditions Kimé.

HEIDEGGER, M. (1984). Sentieri interrotti. Firenze: La Nuova Italia.

IACOMINI, M. (2008). Le parole e le immagini. Saggio su Michel Foucault. Macerata: Quodlibet Studio.

MERLEAU-PONTY, M. (1964). Le visible et l'invisible. Paris: Gallimard.

REDAELLI, E. (2011). L'incanto del dispositivo. Foucault dalla microfisica alla semiotica del potere. Pisa: ETS.

SINI, C. (1978). "Il problema del segno in Husserl e Peirce". Quaderni della Biblioteca Filosofica di Torino 57, 543-558.

TAYLOR, J. R. (1995). Linguistic categorization: prototypes in linguistic theory. Oxford: Clarendon Press.

TOMMASI, W. (1984). Maurice Blanchot e la parola errante. Verona: Bertani Editore.

VIOLI, P. (1997). Significato ed esperienza. Milano: Bompiani. 
Recibido el 2 de marzo de 2020.

Aceptado el 15 de abril de 2020. 